\title{
Cosmic Rays Report from the Structure of Space
}

\author{
A. Annila ${ }^{1,2}$ \\ ${ }^{1}$ Department of Physics, University of Helsinki, 00014 Helsinki, Finland \\ ${ }^{2}$ Department of Biosciences, University of Helsinki, 00014 Helsinki, Finland \\ Correspondence should be addressed to A. Annila; arto.annila@helsinki.fi
}

Received 3 June 2015; Revised 14 August 2015; Accepted 24 August 2015

Academic Editor: Alberto J. Castro-Tirado

Copyright (C) 2015 A. Annila. This is an open access article distributed under the Creative Commons Attribution License, which permits unrestricted use, distribution, and reproduction in any medium, provided the original work is properly cited.

\begin{abstract}
Spectrum of cosmic rays follows a broken power law over twelve orders of magnitude. Since ubiquitous power laws are manifestations of the principle of least action, we interpret the spectrum accordingly. Our analysis complies with understanding that low-energy particles originate mostly from rapidly receding sources throughout the cosmos. The flux peaks about proton rest energy whereafter it decreases because fewer and fewer receding sources are energetic enough to provide particles with high enough velocities to compensate for the recessional velocities. Above $10^{15.6} \mathrm{eV}$ the flux from the expanding Universe diminishes below the flux from the nearby nonexpanding part of the Universe. In this spectral feature, known as the "knee," we relate to a distance of about $1.3 \mathrm{Mpc}$ where the gravitational potential tallies the energy density of free space. At higher energies particles decelerate in a dissipative manner to attain thermodynamic balance with the vacuum. At about $10^{17.2} \mathrm{eV}$ a distinct dissipative mechanism opens up for protons to slow down by electron-positron pair production. At about $10^{19.6} \mathrm{eV}$ a more effective mechanism opens up via pion production. All in all, the universal principle discloses that the broad spectrum of cosmic rays probes the structure of space from cosmic distances down to microscopic details.
\end{abstract}

\section{Introduction}

Cosmic rays $[1,2]$ impinge on Earth's atmosphere with energies that have been measured over twelve orders of magnitude. The particle flux versus energy, when displayed on the log-log plot, compiles mostly from straight lines; that is, the data follows a broken power law [3-9]. Since the power law is a manifestation of the principle of least action in its original form by De Maupertuis [10-13], we are motivated to use this universal imperative to account for particle propagation from cosmic origins to observatories on Earth.

Not only does the spectrum of cosmic rays display power laws, but also other astronomical observations display scalefree patterns [14]. Furthermore, we are motivated to employ the ubiquitous law of nature because it has helped to interpret propagation of light from Type Ia supernovae [15] as well as to reevaluate some other astronomical observations [16-18]. Since production of particles often couples with emission of light, we find the thermodynamic tenet, where everything depends on everything else, as justifiable approach to analyze also the cosmic ray spectrum.

Today, one century since the discovery of cosmic rays, wealth of data has been acquired about these perplexing particles and understanding has emerged, yet it seems hard to put all pieces of the puzzle together [19-21]. Undoubtedly the principle of least action also fails to exhaust all open questions, but the holistic perspective may provide some new thoughts how to interpret data.

\section{The Least-Time Principle}

The principle of least action in its original form generalizes Fermat's least-time principle for propagation of energy in any form, that is, not only in the form of light. Thus the universal law of nature simply says that evolution, that is, changes of state, will consume difference in energy of any kind in least time [12, 13]. For instance, when a particle with mass $m$ propagates with velocity $\mathbf{v}$ along its least-time path, that 
is, along geodesic, the conservation of quanta requires that a change in kinetic energy $E_{k}=2 K=m v^{2}$ equates changes in scalar $U$ and vector $Q$ potentials; that is,

$$
d_{t} 2 K=-\mathbf{v} \cdot \nabla U+d_{t} Q
$$

where the change $d_{t} 2 K=d_{t} m v^{2}$ in kinetic energy is invariably coupled with changes both in the scalar potential $-\mathbf{v} \cdot \nabla U=\mathbf{v} \cdot m \mathbf{a}$ and in energy $d_{t} Q=v^{2} d_{t} m=v^{2} d_{t} E / c^{2}$ which is dissipated from the system to its surroundings or vice versa. The change in mass $d_{t} m$ is for many changes of state, for example, for chemical reactions, small and even minute; nevertheless, if it is omitted, the account is incomplete and conceptual conundrums will follow. Hence $d_{t} m$ ought to be acknowledged and transcribed to the dissipative change in energy $d_{t} Q$ by the familiar mass-energy equivalence in the vacuum.

The equation of evolution (1) from one state to another can be derived from the notion of probability in statistical mechanics of open system $[13,22]$. It is also easy to recognize, just as De Maupertuis did, that (1) is Newton's 2nd law of motion for a change in momentum $\mathbf{p}=m \mathbf{v}$ in its original complete form when multiplying with velocity $\mathbf{v}$; that is,

$$
\begin{gathered}
\mathbf{F}=d_{t} \mathbf{p} \mid \cdot \mathbf{v}, \\
\mathbf{F} \cdot \mathbf{v}=d_{t}(m \mathbf{v}) \cdot \mathbf{v}=\mathbf{v} \cdot m \mathbf{a}+v^{2} d_{t} m, \\
d_{t} 2 K=-\mathbf{v} \cdot \nabla U+d_{t} \mathrm{Q},
\end{gathered}
$$

where the familiar term ma of acceleration is identified with the gradient of $U$, since $d_{t}=\mathbf{v} \cdot \nabla$, as well as the change in mass $d_{t} m$ with dissipation $d_{t} Q=v^{2} / c^{2} d_{t} E$ using the massenergy equivalence $d m=d E / c^{2}$ for the conversion of bound energy in mass to energy $E$ of freely propagating photons in the vacuum which is characterized by the squared speed of light $c$. So, the least-time imperative is equivalent to Newton's notion of net force as it should. Namely, the least-time path points along the resultant force.

The use of mass-energy equivalence (see (2)) in the context of a seemingly nonrelativistic equation may though raise the eyebrows. Note that here $E=m c^{2}$ for the vacuum is regarded merely as the special form of the general form $2 K=m v^{2}$, known as vis viva [23] in the 18th century, most notably, by du Châtelet, s' Gravesande, Leibniz, and de Maupertuis. Later, when kinetic energy was reduced to $K=(1 / 2) m v^{2}$, the notion narrowed to quantify only changes in the body's motion, thereby ignoring concomitant changes in the surrounding energy landscape. For example, when a particle of cosmic origins slows down by losing energy via dissipative processes, the surrounding vacuum will absorb the emitted energy, and hence its state will change too. In other words, every change in the state of system is coupled with corresponding change in its surrounding energy density $[12,13]$. Thus, any equation of motion is incomplete if it ignores motional changes in the surroundings that are concurrent with changes in the state of a particle. Therefore, we conclude that the general principle of nature applies also to the cosmic rays that propagate along least-time paths through the evolving Universe.
The principle of least action is, of course, widely used in physics; however, it is primarily used in the nondissipative and deterministic form as was devised by Lagrange. In contrast, according to (1), the dissipative evolution from one state to another is a nondeterministic process. An outcome of this nonholonomic process depends not only on the initial state but also on the taken path. History emerges because the motion consumes its driving forces which, in turn, affect the motion and so on. In other words, when variables cannot be separated, the equation of evolution (see (1) and (2)) cannot be solved exactly. Although Maupertuis' form did not meet the onetime expectations of a computable law, there is nothing wrong with it. On the contrary, it accurately accounts for evolving nature.

Undoubtedly our reappraisal of the least-time principle in its old original form prompts many a physicist to query for physical assumptions that underlie the tenet. These grounds are minimal and elementary. Namely, quanta of actions are regarded to embody everything, including the vacuum [24]. Specifically the photon is the quantum of action. Its invariance is quantified by Planck's constant $h=E t$ as the product of photon's energy $E$ and period $t$. The photon, as the most elementary action, is a force carrier that moves energy from the system to its surroundings. For instance, X-rays are emitted when electrons are forced to curve on a path. Additionally, the photon itself will lose energy by shifting to red in quest of attaining energetic balance with its surroundings when propagating from the energy-dense nascent Universe to the energy-sparse contemporary surroundings. In other words, no system can change from one state to another without either absorbing or emitting energy.

Maupertuis' principle does not divide the system to an invariant body of mass propagating along some path. Instead integration of momentum $\mathbf{p}$ (of a body) on its path $\mathbf{x}$ yields the entity known as the action $A=\int \mathbf{p} \cdot d \mathbf{x}=n h$. It sums the system in question from quantum of actions up to a certain integer $n$. This 18th century perception of nature in geometrical terms of geodesic actions was consistent and comprehensive. For instance, the dissipative change in mass (see (2)) did not puzzle Euler who quantified the change in mass as a change in the geodesic curvature of an action's path relative to the straight paths of universal reference, that is, the vacuum. Thus the concept of mass as Euler's characteristic merely quantifies how much rays of light will deviate in the vicinity of a body away from their otherwise straight paths in the free space $[18,25]$. This quality of mass is, of course, contained in general relativity too.

In general, the least-time consumption of free energy results in a sigmoid curve, whose central region dominates on the log-log scale as a power law [13]. Similarly the least-time free energy consumption by several mechanisms over a range of energies yields a series of sigmoid curves that follow each other as consecutive lines on a log-log plot; that is, the data follows a broken power law as the spectrum of cosmic rays.

Since the broken power law characterizes the cosmic ray spectrum, we will employ the universal least-time principle to examine the flux of particles over the entire range of measured energies with the aim of relating spectral features to certain 
mechanisms of free energy consumption. So, we will not provide some specific new results on any particular aspect of cosmic rays; instead we will compile a holistic account on the propagation of particles across cosmic distances down to microscopic details of dissipation.

Of course, many an expert may not be in need of comprehensive interpretation by the general principle of physics but prefers a particular mechanistic explanation for each spectral characteristic. We do not discard mechanisms either but see them only in the service of least-time free energy consumption. By the same token we do not regard the power-law characteristic merely as a phenomenological model but a fundamental consequence.

It is perhaps worth emphasizing that the general principle of least time does not unveil specific mechanisms of energy transfer. It only relates a change in the spectral index with the change from one mechanism to another. Therefore we are only able to associate a distinct change with a particular mechanism based on what is known by previous studies about the onset energy. Of course, it is conceivable that in a given spectral band there are two or more main mechanisms. For example, it has deduced that along with particle production processes toward the end of the spectrum, and also the composition of flux changes [26-28]. We cannot exclude these model-dependent conclusions but prefer to account for the changes in spectral index by the least number of parameters that are needed in our model.

\section{Spectral Characteristics}

For the principle to be universal also in the context of cosmic rays, it ought to make sense of the particle propagation over the entire broad band of energies spanned by the particles of cosmic origins. Moreover, each change in the flux ought to be associated with an onset or ending of free energy consumption by a particular mechanism. So, in the following, we will proceed to relate, step by step, each spectral band to a particular process.

3.1. Stationary Flux. Fine features of the cosmic ray spectrum are customarily exposed so that the flux is presented when multiplied by kinetic energy $E_{k}{ }^{\gamma}$ raised to a power of the spectral index $\gamma$. The conventional choice $\gamma=3$ is motivated, as we will show below, because it corresponds to the flux produced by a steady-state partition of sources. Namely, the evolutionary equation of motion (see (1)) integrates to the familiar virial theorem [29], $2 K+U=0$, in the particular case when the system has already attained a free energy minimum state. At the steady state there is no net dissipation; that is, $d_{t} Q=0$, and hence at thermodynamic balance the least-time paths are stationary.

We find from the equipartition theorem that the steadystate flux must fall as one over the cube of kinetic energy, that is, $F \propto 1 / E_{k}^{3}$, to comply with a constant total flux $I$ of particles integrated over all energies in unit time per unit area originating from a maximum entropy partition $\Phi\left(E_{k} / k_{B} T\right)$ of sources in a constant volume, here simply a sphere $4 \pi r_{o}{ }^{3} / 3$ with radius $r_{o}$, over the density of $n$ states
$g\left(E_{k} / k_{B} T\right) d\left(E_{k} / k_{B} T\right)=4 \pi n^{2} d n=4 \pi E_{k}^{2} d E_{k} /\left(k_{B} T\right)^{3}$ with average energy $k_{B} T$; that is,

$$
\begin{aligned}
I & =\int_{0}^{\infty} \frac{F E_{k} \Phi\left(E_{k} / k_{B} T\right) g\left(E_{k} / k_{B} T\right) d E_{k}}{k_{B} T} \\
& =\int_{0}^{\infty} 4 \pi F \frac{E_{k}^{3}}{\left(k_{B} T\right)^{3}} \Phi\left(\frac{E_{k}}{k_{B} T}\right) d E_{k}
\end{aligned}
$$

is a constant.

So, when the observed flux is multiplied with $E_{k}{ }^{3}$, the published data of several measurements [30-45] are roughly constant in the range from some million gigaelectron volts $(\mathrm{eV})$ to about billion gigaelectron volts (Figure 1).

When $F \cdot E_{k}^{3}$ quantity is steady, it means that the particles within this energy range will arrive to detectors with the same energy, on average, as they have left from their sources. So, we reason that the flux above $10^{15.6} \mathrm{eV}$, known as the "knee," stems from sources which are approximately stationary, and hence these sources of particles must be, by and large, in the local nonexpanding part of the Universe. According to astronomical observations, this realm comprises the Milky Way galaxy and the Andromeda galaxy together with nearby smaller galaxies, that is, the Local Group [46, 47] (Figure 2).

Obviously $F \cdot E_{k}^{3}$ data (Figure 1) are not exactly steady above the "knee," but there are regions, especially higher up in energy, where the flux decreases faster and slower than $F \propto 1 / E_{k}^{3}$. So, there must be other processes within the nonexpanding realm of the Universe to be accounted for, besides variation in the flux, for example, due to velocity dispersion of sources.

3.2. High-Energy Flux. At higher energies, that is, above $10^{17.2} \mathrm{eV}$, known as the "2nd knee," the flux falls clearly more rapidly than $E_{k}^{-3}$. This means, according to the profound principle of nature, that kinetic energy of particles exceeds the source potential in these sparse surroundings where they are detected. The energy difference between the system and its surroundings, that is, the free energy, is consumed so that the high-energy particles will slow down. In other words, the kinetic energy of particles is transferred to the potential energy of the surroundings, that is, to the photon-embodied vacuum $[24,25]$.

Earlier two dissipative mechanisms have been identified [48-51]. The first of them opens up for protons with kinetic energy above $\sim 10^{17.2} \mathrm{eV}$. The spectral index changes because the protons will decelerate by producing pairs of electrons and positrons. We recognize these leptons as quantized actions in the form of "vortices" that materialize from the quantized actions embodying the vacuum due to dissipative perturbations, that is, "turbulence" induced by the highenergy protons (Figure 3). Our geometric description of a particle in terms of a quantized action is merely a consistent consequence of the least-action principle. It integrates any momentum $\mathbf{p}$ over its least-time path $\mathbf{x}$ of propagation to a multiple $n$ of Planck's constant $h$; that is, $\int \mathbf{p} \cdot d \mathbf{x}=n h$ [52]. At even higher energies, above $\sim 10^{19.6} \mathrm{eV}$, known as the GZK cutoff, the other dissipative mechanism opens up for protons to decelerate even more effectively by producing 


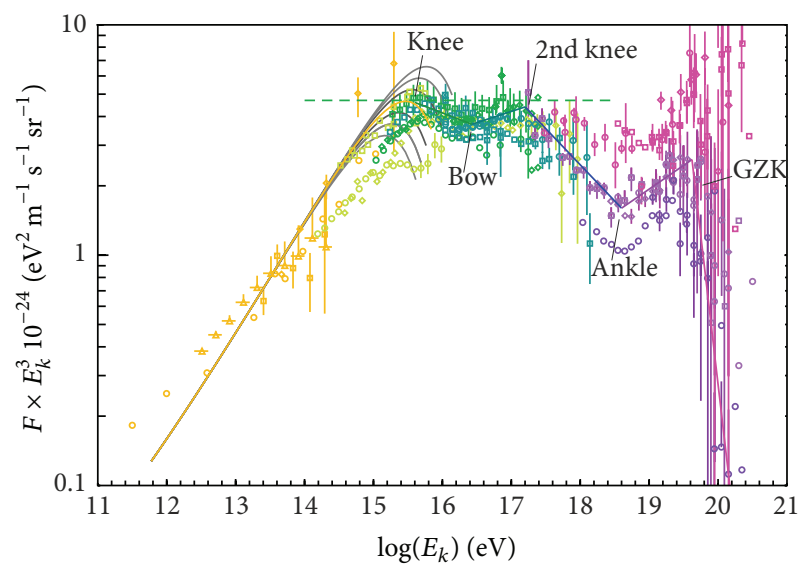

$\begin{array}{ll}\diamond \text { Proton } & \circ \text { Tunka } \\ \triangle \text { Cream } & \square \text { Kascade } \\ \square \text { RUNJOB } & \circ \text { HiRes I } \\ \diamond \text { JACEE } & \square \text { HiRes II } \\ \circ \text { CASAMIA } & \diamond \text { TELE ARRAY } \\ \square \text { Hegra } & \circ \text { Auger } \\ \diamond \text { Akeno } & \circ \text { Haverah } \\ \circ \text { Tibet } & \square \text { AGASA } \\ \square \text { IceTop } & \diamond \text { Yakutsk } \\ \diamond \text { Gamma } & - \text { Computed }\end{array}$

FIGURE 1: Flux of cosmic rays $F$ multiplied by $E_{k}{ }^{3}$ is reproduced from published data (see text for references) without recalibration for unbiased assessment by the principle of least action. Coloring merely serves to emphasize various spectral regions. Comparison of data with the computed flux (orange line) via (5) indicates that most particles below $10^{15.6} \mathrm{eV}$, known as the "knee," have departed from sources in the expanding Universe, whereas above the "knee" the calculated flux from a stationary system with the characteristic $E^{-3}$ (green dashed line) via (3) implies that recorded particles originate from sources in the nearby nonexpanding part of the Universe. The close match of computed flux with data at the "knee" provides an estimate for the radius $r_{o} \approx 1.3 \mathrm{Mpc}$ (with $\pm 10 \%$ uncertainty shown by grey lines) of the nonexpanding part of the Universe. Above the knee the flux decreases as $E^{-3.1}$ because protons will slow down first by producing photons and eventually neutrinos (green line). Then, above $10^{17.2} \mathrm{eV}$, known as the "2nd knee," the flux decreases as $E^{-3.3}$ because protons will slow down more effectively producing pairs of electrons and positrons (blue line), and then above $10^{19.6} \mathrm{eV}$ the flux decreases approximately as $E^{-6}$, known as the GZK cutoff, by the even more effective pion production (purple line). The decelerated protons by various mechanisms will return to lower energies. The reflux from the meson production (violet line) will give rise to the "ankle" at about $10^{18.6} \mathrm{eV}$ above which the flux decreases as $E^{-2.8}$ and likewise the reflux from the lepton production (dark green) will give rise to the "bow" at about $10^{16.4} \mathrm{eV}$ above which the flux decreases as $E^{-2.9}$.

pions. We recognize these mesons as "wavelets" that emerge from turbulence in the surrounding quanta of action $[25,53]$.

We picture the steady-state vacuum as laminar (straight) flows of quanta at the speed of light $[24,25,53]$. The ultrahigh-energy cosmic rays will perturb these uniform and stationary flows to turbulent trajectories that will materialize as particles. This view of a quantized vacuum parallels thoughts about void by the old $a$-tomistic tenet [54]. Since the
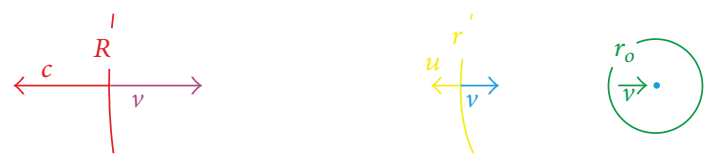

FIGURE 2: Schematic view angle of the Universe opens from a blue dot (at right) that signifies the Earth. Particles that arrive to our detectors with velocity $v$ have departed either with the same velocity (green arrow) from sources in the nearby nonexpanding sphere of the Universe within a radius $r_{o}$ (green circle) or with a higher velocity (blue arrow) from sources that are receding with velocity $u$ as exemplified to reside at a distance $r$ (yellow arc). No particle with velocity $v<c$ will attain our observatories from the perimeter of the Universe at $R$ (red arc) that is expanding with the speed of light $c$.

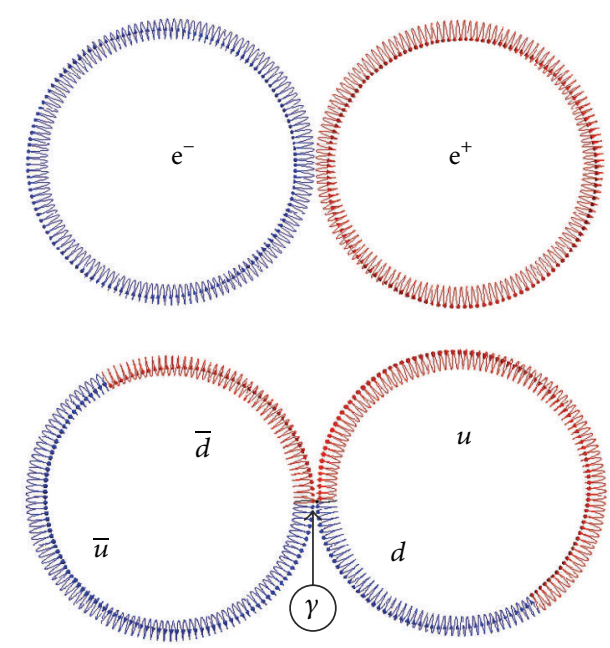

FIGURE 3: When high-energy protons slow down, pairs of electrons $\left(\mathrm{e}^{-}\right)$and positrons $\left(\mathrm{e}^{+}\right)$will materialize from quanta of actions, that is, from photons that embody the vacuum in pairs of opposite polarization. The resulting leptons are described as quantized actions in toroid forms (shown above as color coded by charge). Accordingly, when ultra-high-energy protons decelerate, pions $\pi^{+}$ $(u \bar{d}), \pi^{-}(\bar{u} d)$, and $\pi^{o}(u \bar{u}$ and $d \bar{d})$ will materialize from the photonembodied vacuum. Each of these pseudoscalar mesons, described as a quantized action in a wavelet form, comprises arcs of two quarks $(u, \bar{u}, d, \bar{d})$ joined by a gluon $(\mathrm{g})$, that is, by a high-frequency photon.

leptons and mesons are produced from the massless quanta embodying the vacuum, the proton deceleration by dissipation couples with changes in mass in agreement with (1).

The decelerated protons will, of course, return to lower energies, and hence they will contribute to the flux below the threshold energy of particle production [51]. The proton pile-up is particularly pronounced for the pion production resulting in the "ankle" at $10^{18.6} \mathrm{eV}$, where the reflux of decelerated protons matches the efflux due to less effective electron-positron pair production. In agreement with previous calculations $[48,51]$, we argue by general energetic considerations that the threshold energies and rates of these two dissipative processes are related to each other by the mesonlepton mass ratio $m_{\pi} / m_{e} \approx 265$. This anticipated difference in the threshold energies is readily verified within margin but the relative rates are much more difficult to evaluate 
from the ultra-high-energy data with large uncertainties and apparent difficulties in calibration of datasets with each other (Figure 1).

This perception of the ultra-high-energy data prompts us to reanalyze the range from $10^{15.6}$ to $10^{17.2} \mathrm{eV}$, that is, the flux between the "knees." Admittedly the data as a whole (Figure 1) does not prove unambiguously our insight that the spectral index does change at about $10^{16.4} \mathrm{eV}$, to be known as the "bow," but several sets of data indicate this characteristic. We attribute the increase seen above $E_{k}{ }^{-3}$ reference level in the range from the "bow" to the "2nd knee," as argued above, to the proton pile-up resulting from deceleration by the electron-positron production. The prior decrease below $E_{k}^{-3}$ reference level in the range from the "knee" to the "bow" calls for yet another mechanism of dissipation. So, we propose that the protons slow down by producing neutrinos and quanta of light from the photonembodied vacuum (Figure 4). We regard these dissipative processes plausible because in general changes in particle momentum couple with dissipation of photons, for example, as Bremsstrahlung, as well as with dissipation of neutrinos, for example, from decays processes. Moreover, there is a background of neutrinos in the Universe just as there is the cosmic microwave background radiation. The proposed proton deceleration by the photon and neutrino production is on one hand less effective than $\mathrm{e}^{-} \mathrm{e}^{+}$production but on the other hand the pathway opens up once kinetic energy exceeds potential of the surrounding vacuum and because of this mechanism there is no characteristic threshold energy.

Moreover, we think that it is not a mere coincidence that the proton reflux rate between the "ankle" and the onset of GZK cutoff is comparable to the proton efflux rate between the "2nd knee" and the "ankle" due to deceleration by $\mathrm{e}^{-} \mathrm{e}^{+}$pair production. Namely, $\mathrm{e}^{-} \mathrm{e}^{+}$annihilation as a reverse reaction allows the decelerated protons to adjust their kinetic energy to the surrounding potential. Since the pions themselves decay to electrons and positrons as well as to neutrinos and photons, we reason that the proton reflux rate between the "bow" and the "2nd knee" is comparable to the proton efflux rate between the "knee" and the "bow" due to deceleration by the neutrino and photon production.

The rates of free energy consumption via this diversity of mechanisms, just as the rates of all least-time mechanisms, are proportional to the free energy [13]. Therefore the flux across the energy scale compiles from sections of straight lines when plotted on the log-log scale where intersections of sigmoid curves appear as rounded. The particular parameter values of power law lines (Figure 1, caption) depend on mechanistic details of the particle production that have already been studied by particle physics as well as on the number of sources versus energy that has been screened by astronomy. We cannot obtain these numeric values for cross sections and estimates of sources from the general principle, but we are able to require consistency. For example, the slopes for consecutive energy bands (Figure 1) have been outlined to follow the data so that the reflux of decelerated protons from the electron-positron pair production adds to the flux below its onset energy and accordingly the reflux from the pion

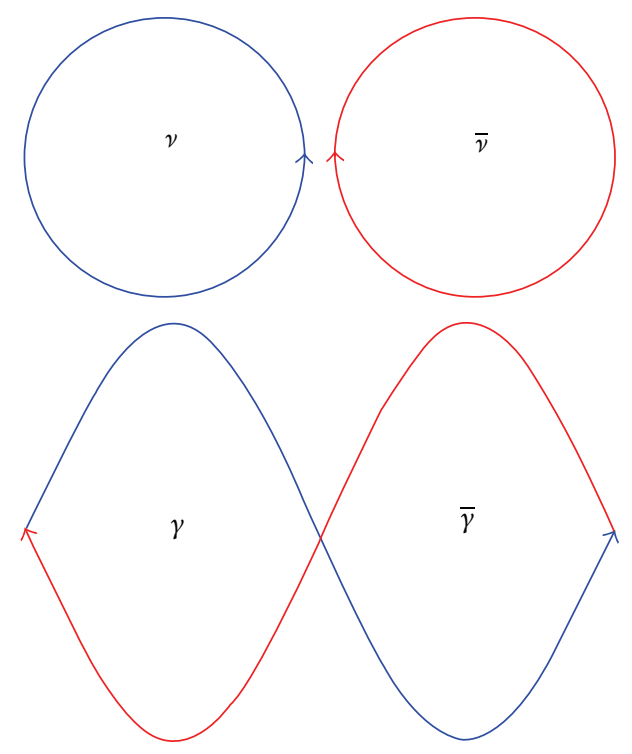

FIGURE 4: When high-energy protons slow down, pairs of neutrinos $(\nu)$ with opposite sense of circulation (color coded) will materialize from quanta of actions, that is, from photons that embody the vacuum in pairs of opposite polarization. The resulting leptons are described as single-quantum actions in circular forms. Additionally, the high-energy protons may decelerate by breaking apart the photon pairs to yield two quanta of light $(\gamma)$ with opposite polarization.

production adds to the flux below its onset. Moreover, the onset energies of electron-positron pair production and the pion production are required to comply with the pion-toelectron mass ratio.

We motivate these constraints for the consecutive bands over extracting a least-square fit, because uncertainties and apparent differences in calibration between experimental datasets are large and because their manipulation would certainly be beyond our competence.

3.3. Flux at Intermediate Energies. At energies below the stationary $E_{k}{ }^{-3}$-region, that is, below $10^{15.6} \mathrm{eV}$, known as the "knee," the flux falls less rapidly (Figure 1). According to the law of nature, this means that the total source potential exceeds the kinetic energy. Therefore the particles must originate also from receding sources in the expanding part of the Universe. Obviously a particle with $E_{k}$ value that is measured at its arrival must have departed at a higher kinetic energy $E_{k u}$ to offset the recessional velocity $u$ of its source. So, although there are more and more receding sources within a larger radius $r$ of integration, fewer and fewer of them are energetic enough to launch particles to high enough speeds for the particles to arrive at $E_{k}$ which is absorbed in detection.

We compute the particle flux arriving at a given kinetic energy $E_{k}$ from the expanding Universe of radius $R=c T$ at its current age $T$ using Hubble's law [55]:

$$
c H=\frac{c}{T}=\frac{c^{2}}{R}=\frac{\left(u-u_{o}\right)^{2}}{r-r_{o}}
$$


by summing up receding sources over the expanding space from $r_{o}$ that defines the radius of nonexpanding space up to $r_{c}$ that corresponds to the maximal recessional velocity $c-v$ of a source that may still deliver the particle to arrive at $E_{k}$; that is,

$$
F \propto \int_{r_{o}}^{r_{c}} \frac{f v}{E_{k u}^{3}} 4 \pi\left(r-r_{o}\right)^{2} d r,
$$

where the arrival rate of particles with velocity $v$ from a source receding with velocity $u$ suffers from Doppler shift $f=v /(u+v)$. We express $E_{k u}{ }^{-3}$ dependence on kinetic energy $E_{k u}=E_{o}\left[\left(1-(u+v)^{2} / c^{2}\right)^{-1 / 2}-1\right]$ at the shell of departure using the familiar Lorentz factor $\gamma=\left(1-v^{2} / c^{2}\right)^{-1 / 2}$ that defines $E_{k}=E_{o}(\gamma-1)$ in terms of the rest energy $E_{o}=m c^{2}$. When $E_{k u}>E_{k}$, the source's recessional velocity $u$ will be compensated so that the particle will arrive with velocity $v=$ $c E_{k} /\left(E_{k}+E_{o}\right)$.

We assume by (5) that the flux is isotropic. This assumption is more and more motivated with decreasing energy, that is, with increasing radius of integration over the sources. Conversely, we expect that the flux would be more and more anisotropic as the radius of integration over the sources narrows with increasing energy, especially above the knee, and ultimately is limited only to our own galaxy. Undoubtedly the flux decreases rapidly not only due to the effective $\mathrm{e}^{-} \mathrm{e}^{+}$ pair and even more effective $\pi$ production but also due to the number of sources diminishing with decreasing limit of integration. This prediction about emerging anisotropy with decreasing number of sources, though, is not fully falsifiable because at ultrahigh energies the number of particles may not be sufficient.

Moreover, it may not be easy to localize the ultrahigh energy sources because in general high-energy fluxes are more susceptible to loss of momentum and concurrent changes in direction than low-energy fluxes. This familiar energy-dependent dispersion manifests itself, for instance, so that blue light refracts more than red. Moreover, the general principle of least time does not say anything about specific mechanisms of dispersal, such as turbulent magnetic fields. These complicated processes facilitate the least-time dispersal of energy from the high-energy sources to the lowenergy surroundings but we cannot calculate their effects on anisotropy. Yet, the proposed tenet is in line with optical observations; namely, radiation is more and more isotropic with decreasing energy.

Numerical integration of (5) carried out by Mathematica [56] closely matches measured data when setting $r_{o} / R=3.2$. $10^{-4}$. The corresponding distance $4.4 \cdot 10^{6}$ ly follows from the estimated age of the Universe $T=R / c=13.8$ billion years. The distance $r_{o}$ is in agreement with astronomical observations that have revealed objects receding when further away than $1.5 \mathrm{Mpc}[46,47]$.

Our computation for the proton flux by (5) reproduces the change in the spectral index at about $10^{15.6} \mathrm{eV}$, where the "knee" is seen in the plot $F \cdot E_{k}^{3}$ versus $E_{k}$ as a hump (Figure 1). We reason that the excess above the horizontal line that designates the stationary-state flux results from those sources which are just barely drifting away from us.
According to the general law this means that energy densities among the bodies, that is, gravitational potential, and the potential in their surroundings, that is, the energy density of free space, are comparable to each other in this intriguing zone of delicate thermodynamic balance $[25,53]$.

Obviously our view of space within $r_{o}$ as stationary is only a guiding approximation, since the nearby sources are actually moving toward us (see (4)) and exhausting their potential energy in producing particles and photons. When departing from the stationary-state approximation we expect the flux to decline above $10^{15.6} \mathrm{eV}$ slightly faster than $E_{k}{ }^{-3}$ in addition to the proposed dissipative mechanism of neutrino and photon production.

3.4. Low-Energy Flux. At even lower energies, for example, below $10^{13} \mathrm{eV}$, the measured flux [57-66] is approximately proportional to $E_{k}^{-2.7}$, which is in agreement with computation by (5) (Figure 5).

The flux increases with decreasing $E_{k}$ because the summation with decreasing velocity $v$ extends to include more and more receding sources with increasing recessional velocity $u$ in the expanding Universe (Figure 2). This conclusion about origins of low-energy particles is in line with observations. Particles with lower and lower energy are more and more likely to originate from extragalactic sources [67]. Conversely, there are point-like high-energy sources in our galaxy [68].

When $E_{k}$ decreases further, the flux levels off toward a maximum, which is according to (4) dictated by the rest energy $E_{o}$. The computed line matches data when $E_{o}$ is set to the proton rest energy. The low-energy end of flux decreases approximately as $E_{k}^{-1}$. When the sources' recessional velocities $u$ tend toward the speed of light $c$, the arrival rate will decline since the particles will suffer more and more from the Doppler shift (Figure 6). Obviously in the limit of a source receding at the speed of light no particle originating from it will arrive to our detectors.

\section{Discussion}

The spectrum of cosmic rays has been also earlier recapped, piece by piece, by power laws. Here we point out that the power-law dependence is not merely a phenomenological model but an inevitable consequence of the least-time free energy consumption. The profound principle allows us to recognize the unity in phenomena that underlie parameters of the broken power law as well as to assign each change in the spectral index to an onset or ending of a process with characteristic energy. Yet, we would like to emphasize that this holistic comprehension of particles' origins and propagation is not exactly new yet insightful in revealing that the cosmic ray spectrum reports from the structure of cosmos in line with other astronomical observations.

The principle of least action is, of course, well established in physics. However, its original form due to Maupertuis used in this study distinguishes from the more familiar Lagrange's form by accounting for dissipative processes, that is, evolution from one state to another. The evolutionary 


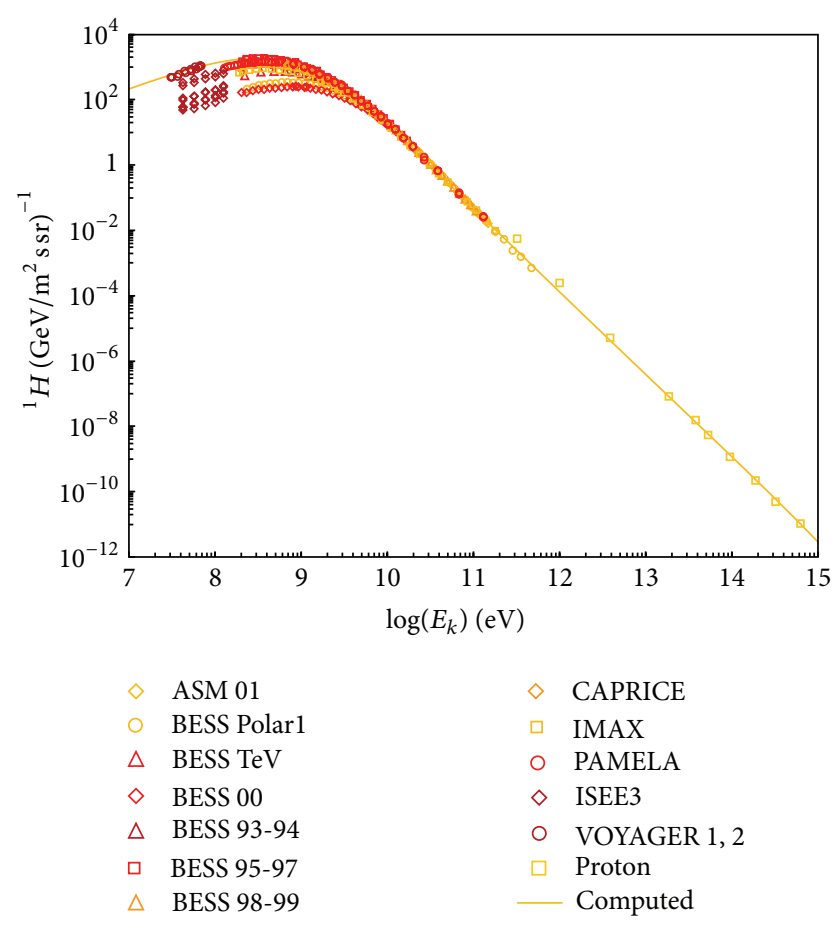

Figure 5: Proton flux $F$ versus energy $E_{k}$ compiled from many measurements, available from Database of Charged Cosmic Rays [79] (see text for references), displays the low-energy spectrum about the flux maximum that is governed by the proton rest energy $E_{o}=m_{p} c^{2} \approx 10^{9} \mathrm{eV}$. The computed line (orange line) has been scaled in energy to match the data to reveal that the proton flux with decreasing energy originates from more and more distant sources in the expanding part of the Universe.

character is necessary to acknowledge the influx of particles from the expanding cosmos as well as to account for the efflux ultrahigh protons and reflux high-energy protons in lepton and meson production processes. Moreover, we find the thermodynamic tenet, where everything depends on everything else, motivated because observations have shown that the average energy density of cosmic rays $\sim 1 \mathrm{eV} / \mathrm{cm}^{3}$ is comparable to those of other energy densities, namely, to that of visible starlight $\sim 0.3 \mathrm{eV} / \mathrm{cm}^{3}$, galactic magnetic field $\sim 0.25 \mathrm{eV} / \mathrm{cm}^{3}$, and cosmic microwave background (CMB) radiation $\sim 0.25 \mathrm{eV} / \mathrm{cm}^{3}[69]$. In other words, the Universe evolves so that any difference in energy will be consumed in least time. This tendency toward balance yields also nearly perfect black body spectrum and uniformity at the largest scale [18].

Nevertheless, many a specialist may at first mistake the adopted thermodynamic tenet as a naïve or dubious method, for instance, when we compute the flux from the expanding Universe without resorting to general relativity. However, the familiar Lorentz factor $\gamma=d t / d \tau=\left(1-v^{2} / c^{2}\right)^{-1 / 2}$ used in the calculation (see (5)) follows from the least-time imperative in the limit of Euclidean continuum, where an infinitesimal change in the kinetic energy $d(2 K) \rightarrow d s^{2}$ equates infinitesimal changes in the potential energy $d U \rightarrow c^{2} d \tau^{2}$ and in the dissipation $d Q \rightarrow c^{2} d t^{2}$ (Figure 7). Additionally,

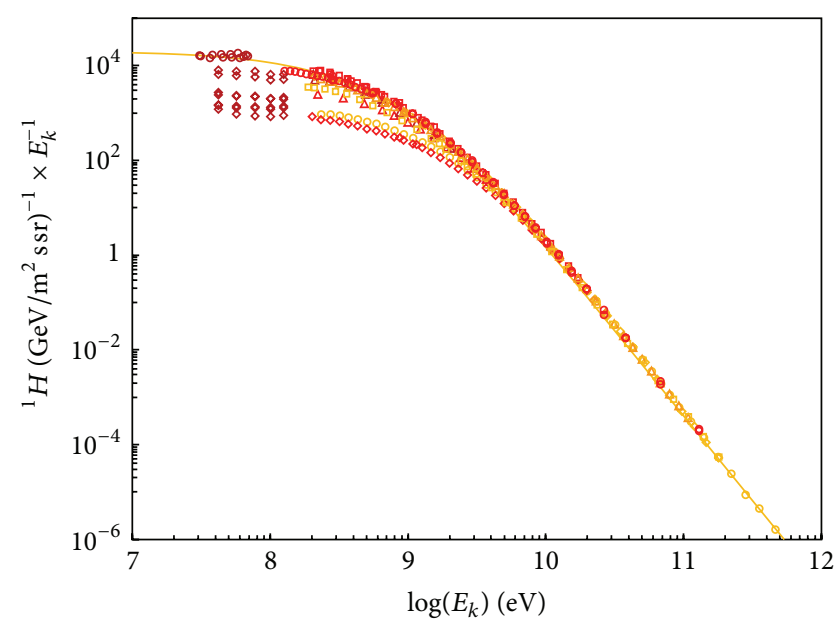

$$
\begin{aligned}
& \text { ASM 01 } \\
& \text { BESS Polar1 } \\
& \text { BESS TeV } \\
& \text { BESS 00 } \\
& \text { BESS 93-94 } \\
& \text { BESS 95-97 } \\
& \text { BESS 98-99 }
\end{aligned}
$$

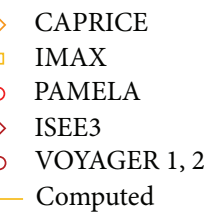

FIgURE 6: Features in the low-energy end of the spectrum are enhanced when proton flux $F$ is divided by energy $E_{k}$. The figure is compiled from many measurements that are available from Database of Charged Cosmic Rays [79] (see text for references). The computed line (orange line) has been scaled in energy to match the data to reveal that the low-energy end of flux originates mostly from rapidly receding sources in the expanding part of the Universe.

as introduced, the relation $E=m c^{2}$, which is often deemed only as relativistic, is regarded by the old principle as a special case of vis viva; that is, $2 K=m v^{2}$. So, our results do not differ dramatically from previous calculations for any particular spectral feature. The only true difference is that we interpret the entire spectrum using one and the same tenet.

Moreover, it may seem curious that we assign the change in the flux known as the "knee" at $10^{15.6} \mathrm{eV}$ to a zone of delicate thermodynamic balance where the gravitational potential due to bodies, for example, galaxies, and the potential contained in the contemporary surrounding vacuum density are comparable to each other. We specifically do not relate the "knee" to any mechanism such as acceleration and propagation of supernova shock waves [70]. However, by the general principle, a force of any kind is nothing but an energy difference per distance in the continuum limit between the system and its surroundings. And hence which way the difference is merely dictates the direction of ensuing motion, that is, to be attractive or repulsive, when the free energy is consumed $[15,17,24]$. At a free energy minimum, there is no net force, and hence those trajectories are stationary. By the same token we are not in need of more sophisticated models than Hubble's law to account for the particles that originate from the expanding Universe. We see the same law to apply likewise also for the contracting (i.e., gravitationally bound) nearby Universe, when explaining anomalies [18, 71, 72]. Hubble's law itself can be understood as an expression of 


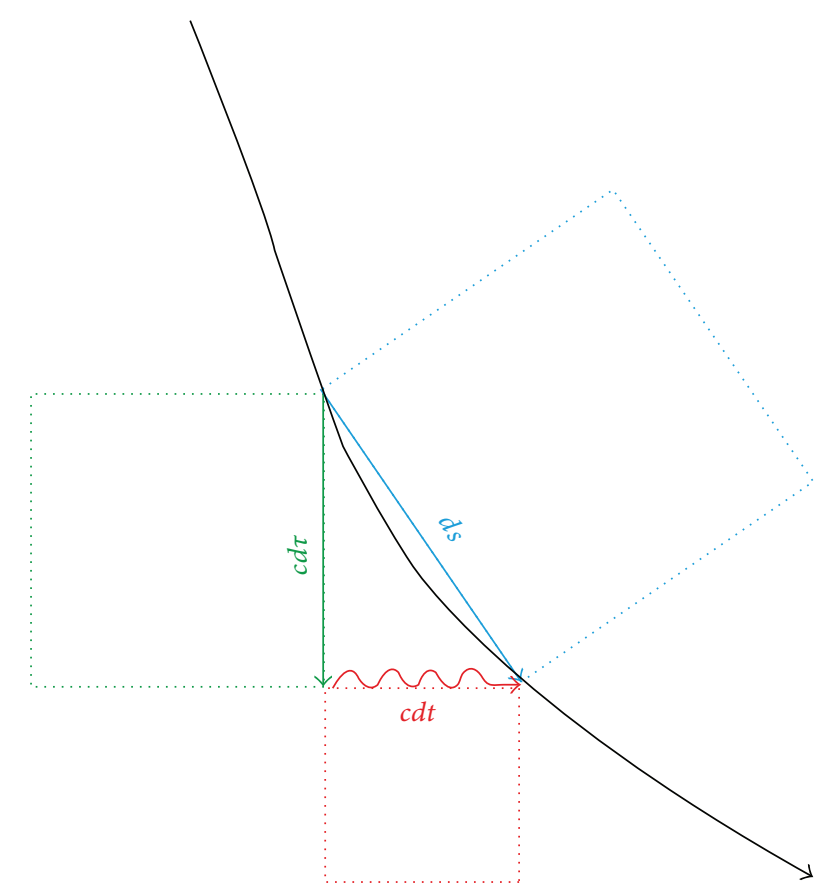

FIGURE 7: According to the principle of least action an infinitesimal change $d s$ (blue arrow) in the kinetic energy $(2 K)$ along the leasttime path (black arrow) equates with an infinitesimal change $c d \tau$ (green arrow) in the scalar potential $(U)$ and infinitesimal change $c d t$ (red arrow) in dissipation $(Q)$. Thus the familiar Lorentz factor $\gamma=d t / d \tau$ follows when the curved least-time path is approximated by straight sections.

the principle of least action (see (1)). It maintains that the acceleration $c H=c^{2} / R=G M / R^{2}$, that is, the equivalence principle, follows from the quest for keeping the energy $M c^{2}$ of the universal background, that is, the vacuum, in balance with the gravitational potential $G M / R$ of the total mass $M$ within radius $R$ of the Universe [24, 73].

The ageing of the Universe and its concomitant decrease in energy density are invariably related to each other when everything is understood as being composed of quantum of actions whose total number $n$ and measure of each $h=E t$ are invariants. In other words, as is stated by Noether's theorem, the integrated action $\forall 2 K d t=n \hbar$ over the Universe is invariant. However, we do understand that the universal imperative (see (5)) does not explicitly expose how the various sources of cosmic rays have evolved in their capacity of expelling particles. We are only able to state that this kind of ageing or other changes that might have accumulated between primordial and contemporary processes, in their capacity to yield particles, will show up as a change in the spectral index of ultra-low-energy flux away from $E^{-1}$ characteristic when such data will be recorded. Moreover, in this low-energy region of the spectrum, we expect flux versus energy to report from the expansion of Universe in the same way as the magnitude versus redshifts of Type Ia supernovae does [15].

Moreover, we emphasize that the principle of least action in its original form supplies us only with scalable functional forms, not with absolute numbers. Additionally, it has been well acknowledged that the cosmic ray data itself, compiled from many measurements over the huge span of energy, is easily subject to miscalibration and uncertainty which compromise evaluation of theoretical explanations.

Our description of the vanishing flux at ultrahigh energies by particle production may seem to parallel earlier accounts, where protons are portrayed to decelerate when interacting with cosmic microwave background (CMB) radiation [4851]. Yet, there is a distinction. Namely, we think that the vacuum is mostly embodied by paired photons, not only by single photons of CMB $[17,24]$. Darkness reigns because electromagnetic fields of the paired quanta cancel each other, yet the quanta themselves prevail in propagation to all directions which gives rise to the vacuum energy density. The vacuum of this nature may seem to resemble renewed ideas about a dynamical vacuum [74] to explain dark energy. However, we do not introduce the photon-embodied vacuum to account for dark energy. In fact, there is no compelling evidence for such a parameter when the data on Type Ia supernovae is interpreted by the principle of least action [15].

According to the old $a$-tomistic tenet, where everything is composed of basic building blocks, that is, the quanta of actions by modern lingo, elementary particles, in this particular case of proton deceleration, electrons, positrons, and pions as well as neutrinos are naturally understood to materialize from the quanta that embody the vacuum in high-energy conditions. Indeed, high-frequency motion in the dynamical Casimir effect produces quanta of light from the seemingly dark vacuum [75]. Perhaps our portrayal on the particle production seems overly pictorial but justified because the same wordings that we use to describe ordinary phenomena are expected to be applied across all scales. The description of particles as quantized actions is more convincingly motivated by the fact that least action paths reproduce measured properties [25].

Currently, how the protons ever acquire ultrahigh energies is somewhat puzzling. Hence, many ongoing studies aim at localizing and characterizing these sources. Magnetic fields of galaxies and supernovae, for example, have been considered already early on as mechanisms of acceleration [76]. The higher the energy is the more anisotropic the flux is for the reason that the sources, which are capable of delivering high-energy particles, are few in numbers and close by. Conversely, the lower the energy is the more isotropic the flux is from numerous sources throughout the Universe. That is to say, we do not discard that the ultrahigh energy data displays signatures of particular mechanisms. Yet, we find it worth reminding, when explaining the whole spectrum by the general physical law, that the mass of a particle, as Euler defined it in terms of geodesic curvature [77], quantifies the geometry of particle's quantized action in relation to the geometry of freely propagating actions that embody the surrounding space, that is, the vacuum [24, 25]. In other words, the mass is not an invariant but depends on the surrounding curvature. So, the protons will strike detectors on Earth with ultrahigh energies, because their mass will increase on the way from the high-density, that is, curved nascent ambiences in an active galactic nucleus, to the lowdensity flat surroundings of our observatories. The same 
effect imposed by the surrounding energy density on particles is witnessed when solar neutrinos climb up in flavor, that is, increase in mass, when they traverse through Earth [78].

In summary, the principle of least action as the supreme law of nature provides a simple yet comprehensive tenet to understand how the broad spectrum of cosmic rays probes the structure of space from its gigantic cosmic spans down to its microscopic details of photon-embodied vacuum.

\section{Conflict of Interests}

The author declares that there is no conflict of interests regarding the publication of this paper.

\section{Acknowledgment}

Dr. Pekka Teerikorpi provided the author with most valuable information and advice.

\section{References}

[1] C. Amsler, K. A. Olive, K. Agashe et al., "Review of cosmic rays," Physics Letters B, vol. 667, pp. 1-1340, 2008.

[2] W. Bietenholz, "The most powerful particles in the Universe: a cosmicsmash," http://arxiv.org/abs/1305.1346.

[3] R. U. Abbasi, T. Abu-Zayyad, J. F. Amman et al., "Observation of the ankle and evidence for a high-energy break in the cosmic ray spectrum," Physics Letters B, vol. 619, no. 3-4, pp. 271-280, 2005.

[4] J. D. Hague, B. R. Becker, M. S. Gold, and J. A. J. Matthews, "Power laws and the cosmic ray energy spectrum," Astroparticle Physics, vol. 27, no. 5, pp. 455-464, 2007.

[5] D. R. Bergman and J. W. Belz, "Cosmic rays: the Second Knee and beyond," Journal of Physics G: Nuclear and Particle Physics, vol. 34, no. 10, pp. R359-R400, 2007.

[6] J. Blümer, R. Engel, and J. R. Hörandel, "Cosmic rays from the knee to the highest energies," Progress in Particle and Nuclear Physics, vol. 63, no. 2, pp. 293-338, 2009.

[7] P. Sokolsky, "Observation of the GZK cutoff by the HiRes experiment," Nuclear Physics B, vol. 196, pp. 67-73, 2009.

[8] G. Matthiae, "The cosmic ray energy spectrum as measured using the Pierre Auger Observatory," New Journal of Physics, vol. 12, Article ID 075009, 2010.

[9] T. K. Gaisser, T. Stanev, and S. Tilav, "Cosmic ray energy spectrum from measurements of air showers," Frontiers of Physics, vol. 8, no. 6, pp. 748-758, 2013.

[10] P.-L. M. De Maupertuis, "Accord de différentes lois de la nature qui avaient jusqu'ici paru incompatibles," in Mémoires de l'Académie Royale des Sciences de Paris, pp. 417-426, 1744.

[11] P.-L. M. De Maupertuis, "Les loix du mouvement et du repos déduites d'un principe metaphysique," in Histoire de l'Académie Royale des Sciences et des Belles-Lettres de Berlin, pp. 267-294, 1746.

[12] P. Tuisku, T. K. Pernu, and A. Annila, "In the light of time," Proceedings of The Royal Society of London A: Mathematical, Physical and Engineering Sciences, vol. 465, no. 2104, pp. 11731198, 2009.

[13] T. Mäkelä and A. Annila, "Natural patterns of energy dispersal," Physics of Life Reviews, vol. 7, no. 4, pp. 477-498, 2010.
[14] Y. Baryshev and P. Teerikorpi, Discovery of Cosmic Fractals, World Scientific World Scientific Publishing, Singapore, 2002.

[15] A. Annila, "Least-time paths of light," Monthly Notices of the Royal Astronomical Society, vol. 416, no. 4, pp. 2944-2948, 2011.

[16] M. Koskela and A. Annila, "Least-action perihelion precession," Monthly Notices of the Royal Astronomical Society, vol. 417, no. 3, pp. 1742-1746, 2011.

[17] A. Annila, "Probing Mach's principle," Monthly Notices of the Royal Astronomical Society, vol. 423, no. 2, pp. 1973-1977, 2012.

[18] A. Annila, "Space, time and machines," International Journal of Theoretical and Mathematical Physics, vol. 2, no. 3, pp. 16-32, 2012.

[19] R. Blandford, P. Simeon, and Y. Yuan, "Cosmic ray origins: an introduction," Nuclear Physics B-Proceedings Supplements, vol. 256-257, pp. 9-22, 2014.

[20] P. Blasi, "Recent developments in cosmic ray physics," Nuclear Physics B-Proceedings Supplements, vol. 256-257, pp. 36-47, 2014.

[21] A. M. Taylor, "UHECR composition models," Astroparticle Physics, vol. 54, pp. 48-53, 2014.

[22] V. Sharma and A. Annila, "Natural process-natural selection," Biophysical Chemistry, vol. 127, no. 1-2, pp. 123-128, 2007.

[23] G. W. Leibniz, "Brevis demonstratio erroris memorabilis Cartesii et aliorum circa legem naturae in conservatione quantitatis motus," Acta Eruditorum Lipsiensibus, pp. 161-163, 1686.

[24] A. Annila, “The substance of gravity," Physics Essays, vol. 28, no. 2, pp. 208-218, 2015.

[25] A. Annila, "The meaning of mass," International Journal of Theoretical and Mathematical Physics, vol. 2, no. 4, pp. 67-78, 2012.

[26] J. Abraham, P. Abreu, M. Aglietta et al., "Measurement of the depth of maximum of extensive air showers above $10^{18} \mathrm{eV}$," Physical Review Letters, vol. 104, Article ID 091101, 2010.

[27] R. Aloisio, V. Berezinsky, and P. Blasi, "Ultra high energy cosmic rays: implications of Auger data for source spectra and chemical composition," Journal of Cosmology and Astroparticle Physics, vol. 2014, no. 10, article 020, 2014.

[28] A. A. Watson, "High-energy cosmic rays and the GreisenZatsepin-Kuz'min effect," Reports on Progress in Physics, vol. 77, no. 3, Article ID 036901, 2014.

[29] R. J. E. Clausius, "On a mechanical theorem applicable to heat," Philosophical Magazine Series 4, vol. 40, pp. 122-127, 1870.

[30] N. L. Grigorov, V. E. Nesterov, I. D. Rapoport et al., "Studies of the energy spectra of high and ultra-high primary cosmic ray particles on the 'Proton' space stations,' Yadernaya Fizika, vol. 11, pp. 1058-1069, 1970.

[31] M. A. Lawrence, R. J. O. Reid, and A. A. Watson, "The cosmic ray energy spectrum above $4^{*} 10^{17} \mathrm{eV}$ as measured by the Haverah Park array," Journal of Physics G: Nuclear and Particle Physics, vol. 17, pp. 733-757, 1991.

[32] M. A. K. Glasmacher, M. A. Catanese, M. C. Chantell et al., "The cosmic ray energy spectrum between $10^{14}$ and $10^{16} \mathrm{eV}$," Astroparticle Physics, vol. 10, no. 4, pp. 291-302, 1999.

[33] F. Arqueros, J. Abel Barrio, K. Bernlöhr, H. Bojahr, I. de la Calle Perez, and J. L. C. González, "Energy spectrum and chemical composition of cosmic rays between 0.3 and $10 \mathrm{PeV}$ determined from the Cherenkov-light and charged-particle distributions in air showers," Astronomy \& Astrophysics, vol. 359, no. 2, pp. 682694, 2000. 
[34] M. Takeda, N. Sakaki, K. Honda et al., "Energy determination in the Akeno Giant Air Shower Array experiment," Astroparticle Physics, vol. 19, no. 4, pp. 447-462, 2003.

[35] T. Antoni, W. D. Apel, A. F. Badea et al., "KASCADE measurements of energy spectra for elemental groups of cosmic rays: results and open problems," Astroparticle Physics, vol. 24, no. 12, pp. 1-25, 2005.

[36] M. Amenomori, X. J. Bi, D. Chen et al., "The all-particle spectrum of primary cosmic rays in the wide energy range from 1014 to 1017 eV observed with the Tibet-III air-shower array," Astrophysical Journal, vol. 678, no. 2, pp. 1165-1179, 2008.

[37] A. P. Garyaka, R. M. Martirosov, S. V. Ter-Antonyan et al., "An all-particle primary energy spectrum in the 3-200 PeV energy range," Journal of Physics G: Nuclear and Particle Physics, vol. 35, no. 11, Article ID 115201, 2008.

[38] R. U. Abbasi, T. Abu-Zayyad, M. Allen et al., "First observation of the Greisen-Zatsepin-Kuzmin suppression," Physical Review Letters, vol. 100, no. 10, Article ID 101101, 5 pages, 2008.

[39] J. Abraham, P. Abreu, M. Aglietta et al., "Observation of the suppression of the flux of cosmic rays above $4 \times 10^{19} \mathrm{eV}$," Physical Review Letters, vol. 101, Article ID 061101, 2008.

[40] R. U. Abbasi, T. Abu-Zayyad, M. Al-Seady et al., "Measurement of the flux of ultra high energy cosmic rays by the stereo technique," Astroparticle Physics, vol. 32, no. 1, pp. 53-60, 2009.

[41] Y. S. Yoon, H. S. Ahn, P. S. Allison et al., "Cosmic-ray proton and helium spectra from the first CREAM flight," The Astrophysical Journal, vol. 728, no. 2, pp. 122-130, 2011.

[42] W. D. Apel, J. C. Arteaga-Velázquez, K. Bekk et al., “The spectrum of high-energy cosmic rays measured with KASCADEGrande," Astroparticle Physics, vol. 36, no. 1, pp. 183-194, 2012.

[43] S. F. Berezhnev, D. Besson, N. M. Budnev et al., "The Tunka133 EAS Cherenkov light array: Status of 2011," Nuclear Instruments and Methods in Physics Research Section A: Accelerators, Spectrometers, Detectors and Associated Equipment, vol. 692, pp. 98-105, 2012.

[44] T. Abu-Zayyad, R. Aida, M. Allen et al., "The cosmic ray energy spectrum observed with the surface detector of the telescope array experiment," The Astrophysical Journal Letters, vol. 768, no. 1, article L1, 2013.

[45] R. Abbasi, Y. Abdou, M. Ackermann et al., "IceTop: the surface component of IceCube," Nuclear Instruments and Methods in Physics Research Section A: Accelerators, Spectrometers, Detectors and Associated Equipment, vol. 700, pp. 188-220, 2013.

[46] A. Sandage, "The redshift-distance relation. IX-perturbation of the very nearby velocity field by the mass of the Local Group," The Astrophysical Journal, vol. 307, pp. 1-19, 1986.

[47] P. Teerikorpi, A. D. Chernin, I. D. Karachentsev, and M. J. Valtonen, "Dark energy in the environments of the Local Group, the M 81 group, and the CenA group: the normalized Hubble diagram," Astronomy \& Astrophysics, vol. 483, no. 2, pp. 383387, 2008.

[48] R. A. Berg and C. N. Lindner, "Electron-proton bremsstrahlung," Nuclear Physics, vol. 26, no. 2, pp. 259-279, 1961.

[49] K. Greisen, "End to the cosmic-ray spectrum?" Physical Review Letters, vol. 16, no. 17, pp. 748-750, 1966.

[50] G. T. Zatsepin and V. A. Kuzmin, "Upper limit of the spectrum of cosmic rays," Journal of Experimental and Theoretical Physics, vol. 4, pp. 78-80, 1966.

[51] V. Berezinsky, A. Gazizov, and S. Grigorieva, "On astrophysical solution to ultrahigh energy cosmic rays," Physical Review D, vol. 74, no. 4, Article ID 043005, 2006.
[52] E. Noether, "Invariante variationsprobleme," in Nachrichten von der Gesellschaft der Wissenschaften zu Göttingen. MathematischPhysikalische Klasse, pp. 235-257, 1918, Translation: E. Noether, "Invariant variation problem," in Transport Theory and Statistical Physics, vol. 1, pp. 183-207, 1971.

[53] A. Annila, "All in action," Entropy, vol. 12, no. 11, pp. 2333-2358, 2010.

[54] J. Palmer, "Parmenides," in The Stanford Encyclopedia of Philosophy, E. N. Zalta, Ed., CSLI, 2008, http://plato.stanford.edu/ entries/parmenides/.

[55] E. Hubble, "A relation between distance and radial velocity among extra-galactic nebulae," Proceedings of the National Academy of Sciences, vol. 15, no. 3, pp. 168-173, 1929.

[56] Wolfram Mathematica, 2008, http://www.wolfram.com/mathematica/.

[57] E. S. Seo, F. B. Mcdonald, N. Lal, and W. R. Webber, "Study of cosmic-ray H and He isotopes AT 23 AU," The Astrophysical Journal, vol. 432, no. 2, pp. 656-664, 1994.

[58] M. Boezio, P. Carlson, T. Francke et al., "The cosmic-ray proton and helium spectra between 0.4 and $200 \mathrm{GV}$," Astrophysical Journal, vol. 518, no. 1, pp. 457-472, 1999.

[59] J. Alcaraz, B. Alpat, G. Ambrosi et al., "Cosmic protons," Physics Letters B, vol. 490, pp. 27-35, 2000.

[60] W. Menn, M. Hof, O. Reimer et al., "The absolute flux of protons and helium at the top of the atmosphere using IMAX," Astrophysical Journal, vol. 533, no. 1, pp. 281-297, 2000.

[61] J. Z. Wang, E. S. Seo, K. Anraku et al., "Measurement of cosmicray hydrogen and helium and their isotopic composition with the BESS experiment," Astrophysical Journal, vol. 564, no. 1, pp. 244-259, 2002.

[62] Z. D. Myers, E. S. Seo, J. Z. Wang et al., "Cosmic ray ${ }^{1} \mathrm{H}$ and ${ }^{2} \mathrm{H}$ spectra from BESS 98," Advances in Space Research, vol. 35, no. 1, pp. 151-155, 2005.

[63] Y. Shikaze, S. Haino, K. Abe et al., "Measurements of 0.2$20 \mathrm{GeV} / \mathrm{n}$ cosmic-ray proton and helium spectra from 1997 through 2002 with the BESS spectrometer," Astroparticle Physics, vol. 28, no. 1, pp. 154-167, 2007.

[64] K. Abe, H. Fuke, S. Haino et al., "Measurement of the cosmicray low-energy antiproton spectrum with the first BESS-Polar Antarctic flight," Physics Letters B, vol. 670, no. 2, pp. 103-108, 2008.

[65] K. C. Kim, K. Abe, H. Fuke et al., "Cosmic ray ${ }^{2} \mathrm{H} /{ }^{1} \mathrm{H}$ ratio measured from BESS in 2000 during solar maximum," Advances in Space Research, vol. 51, no. 2, pp. 234-237, 2013.

[66] O. Adriani, G. Barbarino, G. A. Bazilevskaya et al., "Measurement of the isotopic composition of hydrogen and helium nuclei in cosmic rays with the PAMELA experiment," The Astrophysical Journal, vol. 770, no. 1, article 2, 9 pages, 2013.

[67] X. Chi and A. W. Wolfendale, "Gamma rays from the magellanic clouds and the origin of cosmic rays," Journal of Physics G: Nuclear and Particle Physics, vol. 19, no. 5, pp. 795-804, 1993.

[68] M. Ackermann, M. Ajello, A. Allafort et al., "Detection of the characteristic pion-decay signature in supernova remnants," Science, vol. 339, no. 6121, pp. 807-811, 2013.

[69] A. Castellina and F. Donato, Planets, Stars and Stellar Systems, T. D. Oswalt, I. S. McLean, H. E. Bond, L. French, P. Kalas, M. Barstow, G. F. Gilmore, and W. Keel, Eds., Springer, Berlin, Germany, 2012.

[70] P. O. Lagage and C. J. Cesarsky, "The maximum energy of cosmic rays accelerated by supernova shocks," Astronomy \& Astrophysics, vol. 125, pp. 249-257, 1983. 
[71] M. E. McCulloch, "Modelling the flyby anomalies using a modification of inertia," Monthly Notices of the Royal Astronomical Society: Letters, vol. 389, no. 1, pp. L57-L60, 2008.

[72] J. L. Rosales, "The Pioneer's acceleration anomaly and Hubble'sconstant," http://arxiv.org/abs/gr-qc/0212019.

[73] R. P. Feynman, F. B. Morinigo, W. G. Wagner, and B. Hatfield, Feynman Lectures on Gravitation, Addison-Wesley, Reading, Mass, USA, 1995.

[74] S. Basilakos and J. Solà, "Effective equation of state for running vacuum: 'Mirage' quintessence and phantom dark energy," Monthly Notices of the Royal Astronomical Society, vol. 437, no. 4, pp. 3331-3342, 2014.

[75] C. M. Wilson, G. Johansson, A. Pourkabirian et al., "Observation of the dynamical Casimir effect in a superconducting circuit," Nature, vol. 479, no. 7373, pp. 376-379, 2011.

[76] E. Fermi, "Galactic magnetic fields and the origin of cosmic radiation," The Astrophysical Journal, vol. 119, pp. 1-6, 1954.

[77] M. P. Do Carmo, Differential Geometry of Curves and Surfaces, Prentice-Hall, Englewood Cliffs, NJ, USA, 1976.

[78] A. Renshaw, K. Abe, Y. Hayato et al., "First indication of terrestrial matter effects on solar neutrino oscillation," Physical Review Letters, vol. 112, no. 9, Article ID 091805, 6 pages, 2014.

[79] D. Maurin, F. Melot, and R. Taillet, "A database of charged cosmic rays," Astronomy \& Astrophysics, vol. 569, article A32, 2014. 

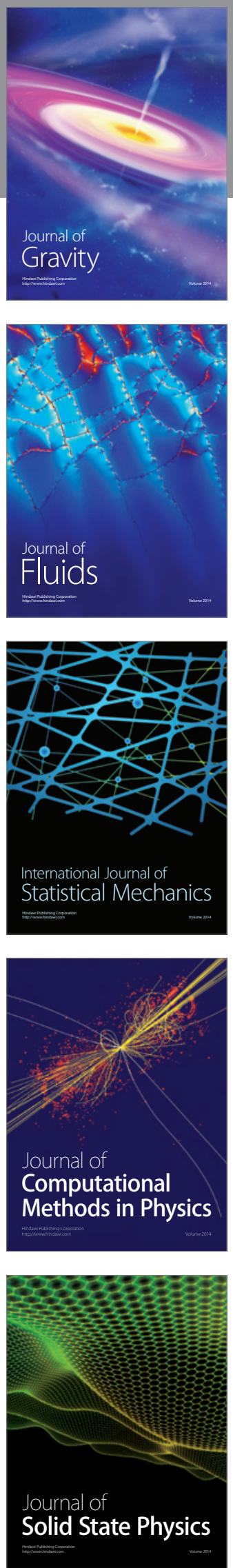

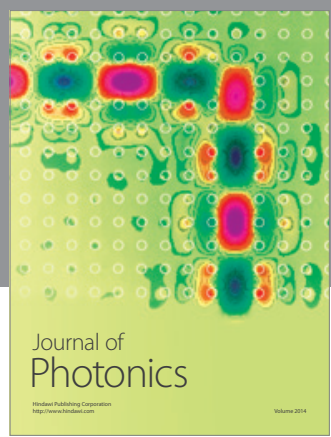

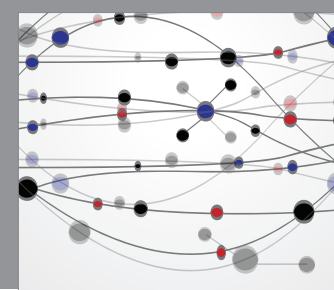

The Scientific World Journal

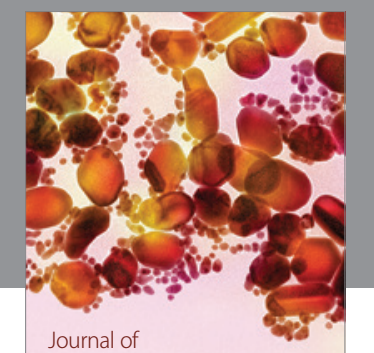

Soft Matter
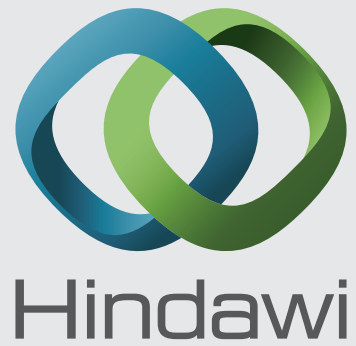

Submit your manuscripts at

http://www.hindawi.com
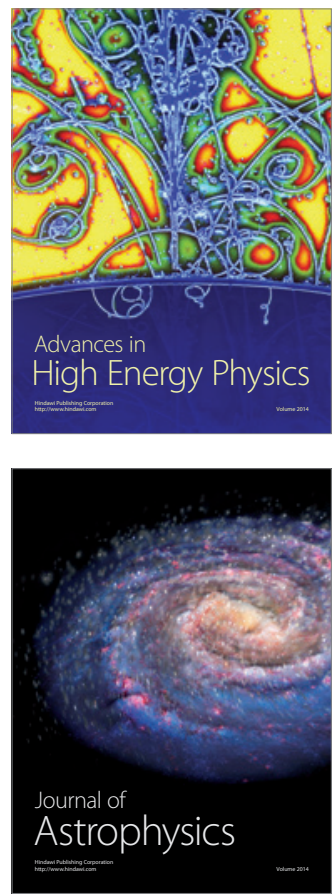
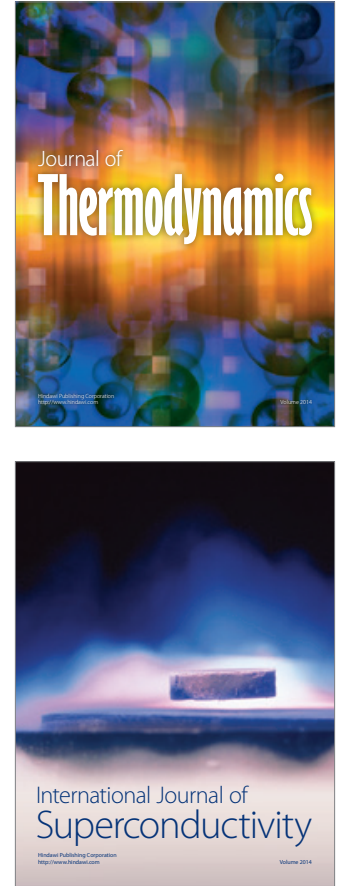
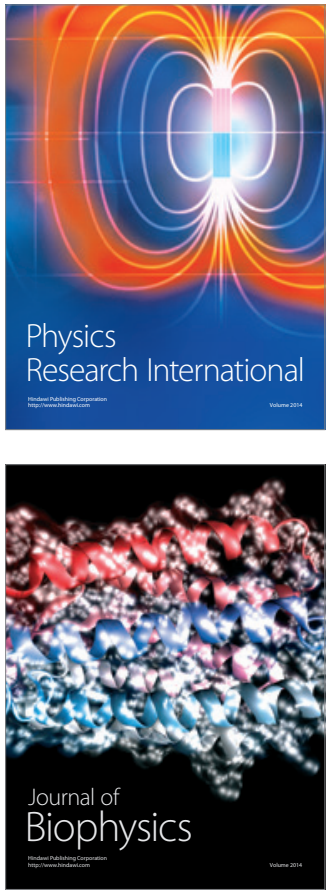
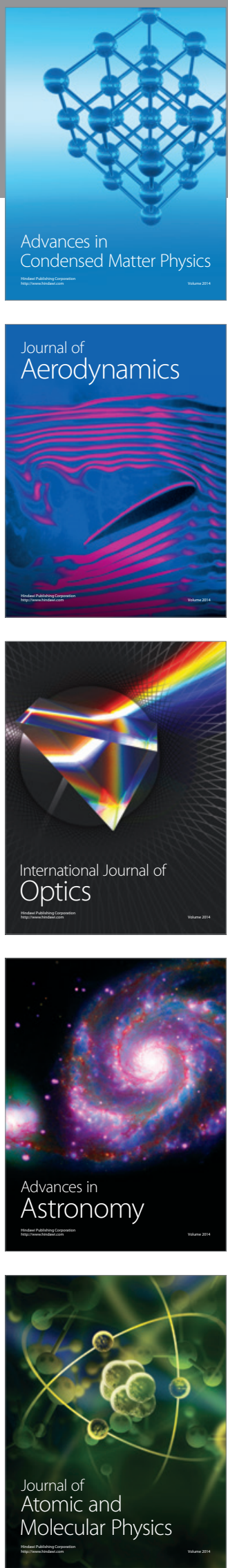